\title{
The effect of the expression of the antiapoptotic BHRF1 gene on the metabolic behavior of a hybridoma cell line
}

\author{
Iván Martínez-Monge ${ }^{1}$, Pere Comas ${ }^{2}$, David Catalán ${ }^{1}$, Jordi Prat ${ }^{2}$, Antoni Casablancas ${ }^{2}$, \\ Carlos Paredes ${ }^{2}$, Martí Lecina ${ }^{3}$, and Jordi Cairo ${ }^{4}$ \\ ${ }^{1}$ Technical University of Denmark \\ ${ }^{2}$ Universitat Autonoma de Barcelona \\ ${ }^{3}$ IQS-Universitat Ramon Llull \\ ${ }^{4}$ Universitat Autònoma de Barcelona
}

April 28, 2020

\begin{abstract}
One of the most important limitations of mammalian cells-based bioprocesses, and particularly hybridoma cell cultures, is the deregulated metabolism related to glucose and glutamine consumption. The high uptake rates of glucose and glutamine (being both the main nutrients used as a carbon, nitrogen and energy sources) yields to the production and accumulation of large amounts of lactate and ammonia in the culture broth. Lactate and/or ammonia accumulation, together with the depletion of the main nutrients are the major causes that triggers the apoptosis in hybridoma cell cultures. The KB26.5 hybridoma cell line producing an IgG3 (used in the ABO blood testing system) was engineered with BHRF1 protein (KB26.5-BHRF1), an EpsteinBarr virus-encoded early protein homologous to the anti-apoptotic protein Bcl-2, with the aim of protecting the cell line from apoptosis. Surprisingly, besides achieving an effective protection from apoptosis, the expression of BHRF1 modified significantly the metabolism of the hybridoma cell line. The comparison of cell physiology and metabolism analysis of the original KB26.5 and KB26.5-BHRF1 revealed an increase of cell growth rate, a reduction of glucose and glutamine consumption, as well as a decrease on lactate secretion in KB26.5-BHRF1 cells. The flux balance analysis allowed quantifying intracellular fluxes of both cell lines. The main metabolic differences were identified in the glucose consumption and, consequently, the lactate generation. The lactate production flux was reduced by $60 \%$ since the need for NADH regeneration in the cytoplasm decreased due to the glucose uptake reduction by more than $50 \%$. In general terms, BHRF1 engineered cell line showed a more efficient metabolism yielding to an increase of the biomass volumetric productivity under identical culture conditions.
\end{abstract}

\section{KEYWORDS}

Hybridoma, genome-scale metabolic model, antiapoptotic gene, mitochondrial transport, BHRF1

\section{1.- INTRODUCTION}

The demand for monoclonal antibodies has severally increased over the last years, mainly for the new applications in therapy, but also for clinical diagnosis and highly specific purification processes (Walsh, 2018; Grilo and Mantalaris, 2019). In this regard, the capacity of mammalian cells to perform complex posttranslational modifications to yield biologically active proteins has led them to be the preferred system for biopharmaceuticals production. About $70-80 \%$ of all biopharmaceuticals, including monoclonal antibodies, viral vaccines and gene therapy vectors, are produced with mammalian cells; and among the top ten selling protein biopharmaceuticals in 2014, six of them are antibodies or antibody-derived proteins. It is therefore not surprising that monoclonal antibodies-based drugs production using mammalian cell-based systems in the 2016 reached almost the double of the 2010 value (Estes and Melville, 2013; Walsh, 2018). In the last years, monoclonal antibodies industrial manufacturing has been based on mammalian cell lines such as 
hybridoma among others (Wurm, 2004; Coco-Martin and Harmsen, 2008; Estes and Melville, 2013; Hnasko and Stanker, 2015).

Mammalian cell-based processes present an important culture limitation: the accumulation of metabolic by-products (i.e. lactate and ammonia) up to cytotoxic concentration, as well as the depletion of essential nutrients, triggers the apoptosis (programmed cell death) (Arden and Betenbaugh, 2004). The prevention of apoptosis during the cell growth has a critical effect on final process productivity: The increase of cell lifespan results in an increase of product of interest synthesis and accumulation, since cells remain productive for a longer time, even after the exponential cell growth phase (Zhang et al., 2018). Moreover, more robust cell lines less sensible to apoptosis allow designing high cell density culture strategies based on keeping low nutrients concentration in narrower ranges (Casablancas et al., 2013).

In the last years, the strategies to generate stress-resistant cell lines preventing apoptosis have been focused on blocking the apoptotic transduction pathways (Smolke, 2010; Henry et al., 2020). Although there are different pathways controlling the activation of signaling cascades of cell apoptosis activation, many of the apoptosis signals converge on the mitochondria, which stores numerous molecules that activate apoptosis (Arden and Betenbaugh, 2004). The most used strategy to prevent apoptosis has been the overexpression of bcl-2 or bcl-xL genes, which inhibits the release of pro-apoptotic molecules from the mitochondria (Smolke, 2010; Vives et al., 2003b). This strategy has been successfully applied in different mammalian cell lines, as CHO or hybridoma, showing higher viabilities and improved robustness in cell culture (Fussenegger et al., 2000; Mastrangelo et al., 2000; Simpson et al., 1997; Tey et al., 2000). The expression of mcl-1, another antiapoptotic gene with similar mechanism to bcl-2/bcl-xL, has also shown good results (Majors et al., 2009). Another approach has been targeting directly the caspase cascade, expressing X-linked inhibitor of apoptosis (XIAP) or cytokine response modifier CrmA, which both act inhibiting directly the caspases (Sauerwald et al., 2003). The expression of different viral proteins has also been reported to have antiapoptotic effects in hybridoma cell cultures, as ksblc-2 from Karposi's sarcoma-associated herpesvirus (Vives et al., 2003a) and bhrf-1 from Epstein-Barr virus (Juanola et al., 2009). Furthermore, the downregulation of the pro-apoptotic genes Bak, Bax and Casp3 has shown to reduce the apoptosis in CHO cells (Xiong et al., 2019).

Additionally, anti-apoptotic genes have shown an effect on metabolism, although this is not fully understood yet (Dorai et al., 2009; Templeton et al., 2014). This is remarkable as cell-based processes present an important limitation regarding the metabolism: deregulated substrates uptake (high consumption rates of mainly glucose and glutamine), what is linked to the secretion and accumulation of lactate and ammonia as by-products of the metabolism (Martínez-Monge et al., 2018a).

The reduction of the secretion and accumulation of lactate remains a hot topic for biomanufacturing industry. Many different approaches have been explored to reduce or delay lactate generation in cell culture including media design by substitution of glucose for alternative carbon sources like fructose or galactose (Altamirano et al., 2006), different fed-batch strategies limiting glucose concentration (Casablancas et al., 2013; Zhang et al., 2004) and several cell engineering approaches as the expression of pyruvate carboxylase (Henry and Durocher, 2011) and downregulation of lactate dehydrogenase (Chen et al., 2001). In all the different scenarios described, a reduction of lactate accumulation up to a certain extend was observed but never totally depleted.

In the present work, a murine hybridoma cell line (KB-26.5) has been engineered to over-express BHRF1 protein (KB26.5-BHRF1). Besides the protective effect against apoptosis achieved, the over-expression of BHRF-1 had an unexpected direct effect on cell physiology and metabolism. Mainly higher cell growth rate and more efficient nutrient usage were observed in batch cultures, significantly reducing the lactate generation. Therefore, metabolic flux balances techniques were applied to better understand the interactions and effects of BHRF-1 protein expression with cell metabolism. The interpretation of the intracellular fluxes obtained allowed an understanding of their effects on the improved cell metabolism and also allowed to arise some hypothesis about the possible BHRF-1 interactions with the metabolic pathways. Nowadays, prevention of apoptosis remains as a relevant research topic as it has been addressed in a recent review by Henry et al., (2020), in which the effect of different anti-apoptotic strategies applied in the last years were presented and discussed. 


\section{2.- MATERIALS AND METHODS}

\section{1.- Cell lines and cell maintenance}

The murine hybridoma cell line used in this work is the KB26.5 which produces an IgG3 antibody against antigen A1 of human red blood cells (used for ABO group determining in blood tests). KB26.5 cell line was transfected with BHRF1 (BamHI rightward reading frame 1)-enconding vector as described by Juanola et al. (2009).

Both cells lines were maintained in $125 \mathrm{~mL}$ polycarbonate shake flask (Corning Inc.) with a working volume of $20 \mathrm{~mL}$ incubated in a $5 \% \mathrm{CO}_{2}$ air mixture and in a humidified atmosphere at $37^{\circ} \mathrm{C}$ (Steri-cult 2000 Incubator). Flasks were continuously agitated at $110 \mathrm{rpm}$ in an orbital shaking platform (Stuart SSL110). Cell passaging was performed every 2 or 3 days at an initial seeding density of $1.5 \cdot 10^{5}$ cells $/ \mathrm{mL}$.

\section{2.- Culture medium}

The basal medium used was Dulbecco's modified Eagle's media (DMEN) (D5796, Sigma) containing 25 mM glucose and $4 \mathrm{mM}$ glutamine. Basal media was further supplemented with $2 \mathrm{mM}$ glutamine (Sigma), $5 \%$ FBS (v/v) (Sigma), $0.5 \%$ Antifoam C Emulsion (Sigma) and $2 \%$ Kolliphorß P 188 (Sigma).

\section{3.- Shake-flasks culturing platform}

250-mL polycarbonate shake-flask (Corning Inc.) with 50-mL of working volume were used for preliminary experiments. Identical conditions to those described under "cell maintenance" were maintained. The seeding density was about $3.0 \cdot 10^{5}$ cells $/ \mathrm{mL}$ and the sampling volume was $1 \mathrm{~mL}$.

\section{4.- Bioreactor and operational conditions}

Bioreactor cell cultures were run in a 2-L Biostat Bplus (Sartorius Stedim Biotech). The bioreactor was equipped with the necessary probes to maintain the desired culture conditions regarding $\mathrm{pH}, \mathrm{pO}_{2}$ and temperature, stirred with two marine impellers at $60 \mathrm{rpm}$. Dissolved oxygen (DO) concentration was measured with an optical probe (VisiFerm DO, Hamilton). DO was set at $40 \%$ of saturation with a constant aeration inflow of $0.20 \mathrm{~L} / \mathrm{min}$, with variable composition controlled by a gas mix air/oxygen station. $\mathrm{pH}$ was measured with a standard electrode (EASYFERM PLUS, Hamilton) and maintained at 7.1 through addition of $\mathrm{NaOH} 0.5 \mathrm{M}$ solution when buffer capacity was not enough to compensate culture acidification. $\mathrm{CO}_{2}$ inlet gas enrichment was fixed at $5 \%$ during the entire culture. Temperature was set at $37{ }^{\circ} \mathrm{C}$.

\section{5.-Analytical methods}

\subsection{1.- Cell number}

Cell number was determined by manual counting using a Neubauer hemocytometer and a phase contrast microscope (Nikon eclypse TS100). Cell viability was determined by the trypan blue dye exclusion method (1:1 mixture of a $0.2 \%$ trypan blue (Gibco ThermoFisher Scientific) in phosphate buffer saline and cell sample).

\subsection{2.- Metabolites concentration}

For the metabolite analysis, samples were centrifuged at 3000g for 3 minutes (Spectrafuge) and the pellet was discarded. Daily measurements of centrifuged samples were obtained for glucose, lactate, glutamine, glutamate, ammonia, $\mathrm{K}^{+}, \mathrm{Na}^{+}$and osmolality using the BioProfile 400 (Nova Biomedical, USA).

Prior the amino acid analysis, supernatants were filtrated using 96-well filter plates (AcroPrep Advance, 0.2 $\mu \mathrm{m}$ Supor Short Tip Natural PP, Pall Corporation). Amino acids were quantified using a method based on Valgepea et al. (2017) with the following modifications: amino acids were derivatized in the HPLC autosampler (Dionex Ultimate 3000) and the sample was injected into an AdvanceBio AAA (2.7 um, $4.6 \mathrm{x}$ $100 \mathrm{~mm}$, Agilent PN: 655950-802) with a guard column (AdvanceBio AAA 2.7 um, 4.6 x 5 mm PN: 820750931). The HPLC gradient was 5-22\% B from 0-7 min, 22-23\% B from 7-8 min, 23-30\% B from 8-9 min, $30-40 \%$ B from 9-14 min, 40-50\% B from 14-16 min, 50-55\% B from 16-18 min, 55-100\% B from 18-18.5 
min and kept at $100 \%$ to $20.1 \mathrm{~min}$, decreased to $5 \%$ B until 24 min where chromatography finished. Buffer A was $40 \mathrm{mM} \mathrm{Na}_{2} \mathrm{HPO}_{4}, 0.02 \% \mathrm{NaN}_{3}(\mathrm{w} / \mathrm{v})$ at $\mathrm{pH} 7.8$. Buffer B was $45 \%$ (v/v) acetonitrile, $45 \%$ (v/v) methanol and $10 \%(\mathrm{v} / \mathrm{v})$ water. Flow rate was $1 \mathrm{~mL} / \mathrm{min}$ from $0-18.5 \mathrm{~min}$ and $1.5 \mathrm{~mL} / \mathrm{min}$ from 18.5-22.1 min thereafter $1 \mathrm{~mL} / \mathrm{min}$ to $24 \mathrm{~min}$. Derivatized amino acids were monitored using a fluorescence detector. OPA-derivatized amino acids were detected at 340ex and 450em nm and FMOC-derivatized amino acids at $266 \mathrm{ex}$ and $305 \mathrm{em} \mathrm{nm}$. Quantifications were based on standard curves derived from serial dilutions of an in-house prepared mixed amino acid standard. The upper and lower limits of quantification were 75 and 0.5 $\mu \mathrm{g} / \mathrm{mL}$, respectively. Chromatograms were integrated using Chromeleon ver 7.1.3.

\subsection{3.- Product concentration}

Product concentration was measured using Valita@ TITER Assay at the moment the cells reached the maximum cell density in bioreactor. Valita $\mathbb{B}$ TITER Assay is a fluorescence polarization assay for the quantitative detection of IgG antibody in cell culture. Standard curve was performed using a known concentration IgG standard (Human IgG Isotype Control, ThermoFisher) diluted in the same cell culture media of the real experiments. 8-point standard curve performed by triplicated was used. The Valitacell Evaluation tool software was used for analyzing titer assays.

\section{6.- Oxygen uptake rate (OUR)}

Determination of the oxygen uptake rate (OUR) was performed by applying the dynamic method (Yoon and Konstantinov, 1994; Zhou and $\mathrm{Hu}$, 1994). This method is based on interrupting the air inflow to the bioreactor for few minutes. Then dissolved oxygen concentration decreases due to the respiratory activity of the cells and to the oxygen desorption from the liquid phase to bioreactor headspace. The specific methodology used in this work is detailed somewhere else (Fontova et al., 2018; Lecina et al., 2006; Martínez-Monge et al., 2018b; Martínez-Monge et al., 2019b).

\section{3.- THEORY / CALCULATION}

\section{1.- Estimation of specific consumption and production rates}

The methodology followed to calculate the specific consumption and production rates for the metabolites analyzes is detailed in Martínez-Monge et al. (2019a).

The specific productivity $\left(q_{p}\right)$ for the cells in the process was calculated using the integral of viable cells (ICV) and the product concentration between the first and the last point as presented in Equation 1 . ICV was calculated using the trapezoidal rule with the function trapz included in Matlab 2019a (Mathworks).

$$
q_{P}=\frac{[\text { Product }]_{\text {final }}}{\text { ICV }}
$$

For the volumetric productivity $\left(\mathrm{V}_{\mathrm{P}}\right)$, the final product concentration and the total process time was used as shown inEquation 2 .

$$
V_{P}=\frac{[\text { Product }]_{\text {final }}}{t_{\text {total }}}
$$

\section{2.- Reduced genome-scale metabolic model}

In this study, the reduced genome scale metabolic model published by Quek et al. (2010) was further modified to adapt it to the experimental conditions. The original model is free available in Systems Biology Markup Language (SBML) (Martínez et al., 2013). The model was derived from the complete Hybridoma genomescale metabolic model constructed from a generic genome-scale metabolic model of Mus musculus (Quek and Nielsen, 2008; Sheikh et al., 2005). The adaptation process to obtain a reduced model able to perform flux analysis for the experiments performed in hybridoma is detailed in (Martínez-Monge et al., 2019a). The base metabolic model does not contain the biomass formation equation, since the objective function 
defined was the maximizing or minimizing of the ATP yield. So, the base model was modified by adding 14 reactions involved in the synthesis of all metabolites precursors needed for biomass formation, as detailed in Martínez-Monge et al. (2019a). The final model is composed by 362 internal and 36 external reactions that overall involve 395 metabolites. A complete list of all included reactions and metabolites used is freely available in theSupplementary Data 1 in xlsx format.

\section{3.- Flux Balance Analysis of the reduced model}

Parsimonious Flux Balance Analysis approach (p-FBA) was performed using Optflux 3.4 Software (Rocha et al., 2010), a user-friendly computational tool for metabolic engineering applications. p-FBA is a variant of flux balance analysis that minimizes the total material flow to achieve an objective, while maintaining the optimal growth (Lewis et al., 2010). The graphical representation of the p-FBA was generated using Omix Visualization Software (GmbH\&Co.KG), as described in Droste et al. (Droste et al., 2011), where only the most significant fluxes for this study were represented.

As usual in FBA with genomic models, the system was under-determined and there existed some degrees of freedom. To find the optimal state, p-FBA uses the optimization of a certain objective function, in this case the maximum value of ATP generation (EF0002). Cytoplasmic malic enzyme was considered to be inactive and NADPH required for biosynthesis could only be regenerated by pentose phosphate pathway, as this degree of freedom can only be solved using more advanced fluxomic techniques (R00216 [0 0]) (Quek et al., 2010). Glutaminolysis was constrained to go in the direction of oxoglutarate generation (R00243mitoc [0 10000]) (McKeehan, 1986). Specific consumption/production rates of experimental metabolites measured were added to the model using the mean of the duplicates \pm standard deviation.

\section{RESULTS AND DISCUSSION}

\section{1- Effect of BHRF-1 on cell physiology}

4.1.1- Cultures with non-controlled $\mathrm{pH}$ and $\mathrm{pO}_{2}$ conditions: Shake flasks cultures

After engineering KB26.5 hybridoma cell lines with BHRF-1, the transformed cell population was kept under selective pressure for several passages. Protective effect of BHRF1 against apoptosis was studied and described previous to the present work (Juanola et al., 2009). Then, differences in terms of cell physiology between the parental cell line KB26.5 and the engineered KB26.5-BHRF1 were observed after selecting the engineered cell population. The main physiological parameters were initially determined in shake flask cultures. Time profiles for cell growth, cell viability, glucose and lactate concentrations for both cell lines (A1 for KB26.5 and B1 for KB26.5-BHRF1) are displayed in Figure 1 . Napierian logarithm of viable cells versus time is plotted to better understand the changes in cell growth rate, as it will be discussed further down (A2 for KB26.5 and B2 for KB26.5-BHRF1). Considering that the experiments were carried out in parallel by duplicate (same time points), the mean values are plotted together with the standard deviation.

The parental KB26.5 cell line reached a cell density about $1.79 \pm 0.3 \cdot 10^{6}$ cells/mL after 72 hours of culture, when cell viability started to decrease sharply. Concerning glucose and lactate concentration, high consumption and production rates respectively could be deduced by the sharp decay of the concentration profiles. Parallel to "plateau" of the cell growth profile, glucose consumption and lactate production also indicates a metabolic arrest at $72 \mathrm{~h}$ of culture, since their concentration values become almost constant. Cell viability decays drastically right after the cell density peak (maximum cell density), reaching values about $50 \%$ at 96 hours (24 hours after the cell density peak).

The growth, viability, glucose and lactate profiles obtained from KB26.5-BHRF1 cultures were absolutely different than those for the parental cell line. Cell culture expansion was much faster, and maximum cell density reached was about $3.92 \pm 0.03 \cdot 10^{6} \mathrm{cell} / \mathrm{mL}$, what represents a 2 -fold increase in respect to the parental cell line. Also, very different was the cell viability profile after the cell density peak, since cell viability was kept over $90 \%$ for 24 hours after the maximum of cell density, indicating the delay on the apoptosis due to the effects of BHRF1. Glucose and lactate profiles showed an initial phase of glucose consumption and lactate generation; glucose concentration profile decrease was similar to the obtained in KB26.5 culture, but lactate 
production rates were clearly lower being reduced by almost half. However, since cell density was almost doubled, the specific glucose consumption rate was clearly affected by BHRF1 expression and, consequently, the lactate production. In order to better quantify the differences, Table 1 complies the main physiological parameters for both cell lines.

In general terms, the expression of BHRF1 was beneficious for cell physiology and growth. Cell growth rate was increased by about $78 \%$, but also glucose consumption specific rate was decreased by $15 \%$ and lactate production reduced to more than $50 \%$. Overall resulted in a more efficient usage of the main carbon source. This fact is translated in a more than 2-fold increase in total cell density reached using the same media and culture conditions.

\subsection{2- Bioreactor cultures with controlled $\mathrm{pH}$ and $\mathrm{pO}_{2}$ conditions}

In order to confirm the beneficial effect of BHRF1 on cell physiology (on cell growth and nutrients usage), cultures of both cell lines were performed in 2-Litres bioreactor. Bioreactor cultures should not only discard that the differences encountered before were related to changing culturing conditions when culturing cells in shake flasks, but also to generate enough data to feed the metabolic model and perform the Flux Balance Analysis. In addition, product concentration $\left(\mathrm{IgG}_{3}\right)$ was measured from the moment the cells reached the maximum cell density. Figure 2 shows the time profile of cell density and viability; glucose, lactate and product concentrations evolution. As the experiments were carried out in the same bioreactor by duplicate but not in parallel (different time points), both replicates are plotted for each cell line, showing comparable behaviors. Napierian logarithm of viable cells versus time is plotted for each replicate inFigure $\mathbf{3}$, to better understand the changes in cell growth.

In general terms, the results obtained in 2-Litres bioreactors are consistent with those previously observed in shake flask cultures. Maximum of cell density was slightly improved for KB26.5, reaching values about $2.34 \pm$ $0.39 \cdot 10^{6} \mathrm{cell} / \mathrm{mL}$, probably due to the $\mathrm{pH}$ control. Even tough lactate was produced at similar rates, the lack of $\mathrm{pH}$ control in the shake flaks probably negatively affected cell growth, situation reverted in the bioreactor cultures. In the case of KB26.5-BHRF1, the maximum cell density observed was significantly increased, obtaining $3.93 \pm 0.01 \cdot 10^{6}$ cell $/ \mathrm{mL}$. In addition, the viability strongly decayed after $48 \mathrm{~h}$ for KB26.5, and it was maintained above $90 \%$ the entire culture for BHRF1-KB26.5.

Despite the concordance with the results obtained in shake flask, a difference was observed since glucose was completely depleted in KB26.5 culture, indicating the extension of the culture time due to the pH control, what could have an effect on cell density increase. To further compare the performance of both strains in Bioreactor, main physiological parameters were calculated and complied in Table 2 .

Similarly to shake flask culture results, cell growth was improved by more than $30 \%$ in KB26.5-BHRF1, and also glucose consumption and lactate generation rates were reduced $42 \%$ and $54 \%$ respectively. Therefore, differences between strains should be related to the effects of BHRF1 on cell physiology instead of being a consequence of the culturing conditions. Due to the extension of the growth phase, KB26.5-BHRF1 increased the final product concentration by 3.7 times. The specific and volumetric productivity were also increased by 2 and 3 times respectively.

Finally, and before preforming Flux Balance Analysis (FBA), the specific consumption rates for all the metabolites measured in bioreactor experiments were calculated in the exponential growth phase (Table 3 ). Values have been calculated as the mean of the data obtained by duplicates.

Again, the results coincide in terms of lower specific consumptions rates for glucose and for almost all amino acids, as well as lower production rates of by-products, what might be a signal of a more efficient cell metabolism, somehow mediated by the expression of BHRF1. It should be emphasized the significant reduction in glutamine consumption and, therefore, in the ammonia generation, which is well known to be a toxic by-product in mammalian cell cultures (Yang and Butler, 2000), obtaining a double benefit of lactate and ammonia reduction in culture. Alanine generation was also significantly reduced in KB26.5BHRF, taking into account that is an amino acid that it is often accumulated in mammalian cell cultures 
(Quek et al., 2010). In any case, a metabolic flux balance is required to evaluate and discuss the possible effects of the protein BHRF1 on the general behavior of the engineered line in respect to the parental cell line. Interestingly, the expression of BHRF1 has an effect on such metabolism improving the efficiency of nutrients usage needed for cell growth (see Table 1, 2 and 3 ), the reduction of by-products, as well as, the increase of cell growth.

\section{2- Effect of BHRF1 on cell metabolism: Flux Balance Analysis}

Metabolism of animal cells growing in high glucose concentration media is characterized by their high rates of glycolysis fluxes. Glucose metabolism of aerobic microorganisms yield pyruvate, which is partially converted into acetyl-CoA and then can be completely oxidized to $\mathrm{CO}_{2}$ and $\mathrm{H}_{2} \mathrm{O}$ in TCA cycle. However, in mammalian cell lines, pyruvate is primarily converted into lactate (Martínez-Monge et al., 2018a). Generation and accumulation of large amounts of lactate, since glucose cannot be completely oxidized, is the major consequence of such high glycolytic fluxes, leading to an unbalanced metabolism. This metabolic behavior has been observed in many hybridoma cell lines, regardless of the level of dissolved oxygen in culture. Lactate generation from pyruvate seems to be necessary to fulfill the NADH regeneration requirements in the cytoplasm (Mulukutla et al., 2012), due to the limiting transport rates of NADH into mitochondria, where can also be regenerated. Even though pyruvate conversion to lactate is a much less energetically efficient process than its oxidation in the Krebs cycle (Martínez et al. 2013).

Flux Balance Analysis (FBA) is a usefulness tool to obtain more information about the redistribution of the internal metabolic fluxes and could help to generate some hypothesis about the effects on metabolism generated by BHRF1. Figures 4 and $\mathbf{5}$ show the distribution of the metabolic fluxes using the genomederived model for Mus musculus detailed in the Calculations section. Metabolic flux balances were conducted using the data obtained from the bioreactor cultures.

FBA shows a deregulated metabolism in both cell lines, characterized by high glycolytic fluxes and the consequent lactate generation and secretion. However, glycolytic fluxes were reduced by $54 \%$ (bottom part of glycolysis as a reference) due to the reduction in glucose consumption (51\%) in KB26.5-BHRF1. Lactate generation flux was dropped to more than $60 \%$. Despite the lower fluxes in glycolysis presented by KB26.5BHRF1 strain respect to KB26.5, the rate of carbon influx from cytoplasm to mitochondria through pyruvate was $14 \%$ higher (320 versus $274 \mathrm{nmol} /(\mathrm{mg} \cdot \mathrm{h})$ ). In other words, when calculating the fluxes in mass units (mg metabolite/(mg DCW $\cdot \mathrm{h})$ ) only $13 \%$ of the total glucose consumed is entered to the mitochondria in KB26.5 cells, whereas it increases up to $32 \%$ in the engineered KB26.5-BHRF1. When performing similar calculations to estimate the lactate generation ratio regardless the glucose consumption, lactate formation represents about $87 \%$ and $69 \%$ of the consumed glucose in KB26.5 and KB26.5-BHRF1, respectively.

Lactate generation in mammalian cell cultures is a well-known issue that has been extensively studied (Hartley et al., 2018; Zagari et al., 2013). At present, the most accepted hypothesis of why lactate is generated argues in the regeneration of the reducing power (NADH) in the cytoplasm, due to the high glycolytic fluxes (Hartley et al., 2018; Zheng, 2012). To this end, there are two ways to regenerate NADH into the cytoplasm: 1) pyruvate conversion to lactate and 2) Malate-Aspartate Shuttle (Mulukutla et al., 2012). The interesting point is that Malate-Aspartate Shuttle allows not only to regenerate NADH but also to increase TCA cycle fluxes (importing malate), allowing then to generate energy in form of ATP. On the other side, lactate generation provokes the total loss of both carbon source and ATP generation. Then, the reason for lactate generation could be found in a flux limit of Malate-Aspartate Shuttle, leading the cells to generate lactate and display this wasteful metabolism.

In the glucose breakdown to two pyruvate molecules, two molecules of ATP and two of NADH are generated. Since the inner mitochondrial membrane is impermeable to NADH, Malate-Aspartate Shuttle works as an indirect transport system. It has been reported that the flux through this transport occurs at lower rates than glycolysis (Schantz et al., 1986), and the increased LDH activity is due to the inability of transporting NADH through the shuttle at the same rates in which is generated. Under conditions of increased cellular energy demand, higher glycolytic fluxes are observed and, consequently, NADH production rates increases 
proportionally. Such increase in $\mathrm{NAD}^{+}$regeneration needs is compensated by higher LDH activity in the cytosol, but not differences in the Malate-Aspartate Shuttle fluxes are observed (Robergs et al., 2004).

In this case, FBA shows that the rate of transport of reduction power from cytoplasm to mitochondria through Malate-Aspartate Shuttle seems to be a bit higher in KB26.5-BHRF1 than in KB26.5, showing a $19 \%$ of increase, with a rate of 228 versus $192 \mathrm{nmol} /(\mathrm{mg} \cdot \mathrm{h})$ in the aspartate-glutamate mitochondrial symport transport. However, in both cases the high glycolytic fluxes lead the cells to generate lactate, as the MalateAspartate shuttle seems to not be enough powerful to couple with the cytoplasmic NADH regeneration. In addition, a slight increase in pathways related with the biomass formation is observed in KB26.5-BHRF1, due to the higher growth rate; as Pentose Phosphate Pathway to generate nucleotides or citrate export from the mitochondria to lipids synthesis.

Due to the high growth rate in KB26.5-BHRF1, an increase in the TCA fluxes could be expected, to generate more energy and therefore biomass. However, comparing TCA cycles for both cell lines no significant increase is observed, what is in accordance with the similar oxygen consumption rate (Table $\mathbf{5}$ ). What it is clear from the FBA performed is the evident collapse between glycolysis and TCA pathways in both cases, as the high glucose uptake rate collapses the transport of NADH to mitochondria, thus NADH should be regenerated into the cytoplasm using pyruvate by means of lactate dehydrogenase.

The reduction of the glycolytic pathway in KB26.5-BHRF1 involves a reduction of ATP production in the cytoplasm, as well as a reduction of NADH formation. Consequently, the needs for oxidizing the NADH formed in the cytoplasm also decreased and, together with the slightly higher rates related to MalateAspartate Shuttle, yielded to a reduction of the lactate formation. To further discuss the results, an analysis of the synthesis and consumption of ATP was performed. Figure 6 shows the distribution of the ATP formation (in regard to the reaction in which ATP is generated) and its consumption.

The results show a significant reduction in the total ATP generation and, as a consequence, ATP consumption in KB26.5-BHRF1 compared with the parental cell line. As pointed out above, higher amounts of ATP were synthetized along glycolysis in parental KB26.5 (proportionally to glycolytic fluxes increase), but also an increase in the oxidative phosphorylation pathway is observed, being very important for the discussion for its high capacity of ATP generation. Regarding the ATP consumption, KB26.5-BHRF1 needs more ATP to generate more biomass, which also includes other pathways as lipids and nucleotide synthesis. The most interesting part, and the key fact of this analysis, is the huge difference in ATP requirements for cell maintenance. One possible explanation of this maintenance reduction could be the higher efficient substrate consumption and specially the lactate generation reduction of KB26.5-BHRF1. As recently pointed out by Buchsteiner et al. (2018), the energy requirements in lower lactate production cell lines may decrease due to the lower energy requirements for maintaining ion gradients.

The results presented here suggest that the engineered KB26.5-BHRF1 hybridoma cell line somehow have altered such metabolism. BHRF1 is an antiapoptotic protein located in the inner mitochondrial membrane (Milian et al. 2015). Due to this fact, BHRF1 could be somehow affecting carbon and NADH/NAD+ transport between cytoplasm and mitochondria. In fact, most of the antiapoptotic genes reported in literature are known to be bind at the mitochondrial membrane, regulating the apoptosis through modulation of the mitochondrial permeability, but also playing and important role in the metabolic processes of mitochondria (Majors et al., 2007). Dorai et al. (2009) reported the effect of two antiapoptotic genes in the metabolism of $\mathrm{CHO}$, showing an important reduction in the final lactate concentration due to the lactate consumption during the culture. In addition, engineered cells showed a more efficient nutrient consumption profile and less by-products generation, as ammonia or alanine, as observed in this study.

\section{5.-CONCLUSIONS}

The results presented in this work have evidenced the beneficious effect of antiapoptotic gene BHRF1 in hybridoma cultures in terms of cell growth, mAb productivity and metabolism; characterized by a more efficient usage of nutrients, doubling the cell density in culture under identical conditions and same culture media. The specific productivity of the cells has been multiplied by two, obtaining three times increase of 
final $\mathrm{IgG}_{3}$ concentration.

The study and comparison of the intracellular fluxes by means of the Flux Balance Analysis of the KB26.5 and KB26.5-BHRF1 engineered cell lines has enlightened the interactions and effects of BHRF1 protein on the metabolic pathways. In short, BHRF1 primarily affected glucose uptake rate, reducing glycolysis by $50 \%$ and consequently reducing the lactate generation by more than $60 \%$. Interestingly, the total ATP generation in the engineered KB26.5-BHRF1 cell line has been significantly decreased due to the lower energy requirements for maintenance, probably due to the lower energy requirements for maintaining ion gradients, although this hypothesis should be evaluated with further metabolic flux analysis experiments.

The use of antiapoptotic genes has been extensively explored in the last years, as manifested in a very recent review about the attenuation of apoptosis in CHO cell line (Henry et al., 2020). Many efforts have been displayed to understand how these genes affect cell apoptosis; but the impact of those in protein production and specially in metabolism have not been completely understood by the scientific community. To tackle this issue, a strategy to obtain the isogenic cell lines should be implemented as it would allow the comparison between different anti-apoptotic genes and their effect.

\section{REFERENCES}

1. Altamirano C, Illanes A, Becerra S, Cairó JJ, Gòdia F. 2006. Considerations on the lactate consumption by $\mathrm{CHO}$ cells in the presence of galactose. Journal of Biotechnology $125: 547-556$.

2. Arden N, Betenbaugh MJ. 2004. Life and death in mammalian cell culture: strategies for apoptosis inhibition. Trends in Biotechnology $22: 174-180$.

3. Buchsteiner M, Quek L-E, Gray P, Nielsen LK. 2018. Improving culture performance and antibody production in $\mathrm{CHO}$ cell culture processes by reducing the Warburg effect. Biotechnol. Bioeng.115 $: 2315-2327$.

4. Casablancas A, Gámez X, Lecina M, Solà C, Cairó JJ, Gòdia F. 2013. Comparison of control strategies for fed-batch culture of hybridoma cells based on on-line monitoring of oxygen uptake rate, optical cell density and glucose concentration. Journal of Chemical Technology \& Biotechnology 88 :1680-1689.

5. Chen K, Liu Q, Xie L, Sharp PA, Wang DIC. 2001. Engineering of a mammalian cell line for reduction of lactate formation and high monoclonal antibody production. Biotechnology 83 Bioengineering 72 :55-61.

6. Coco-Martin JM, Harmsen MM. 2008. A review of therapeutic protein expression by mammalian cells. BioProcess International6 :28.

7. Dorai H, Kyung YS, Ellis D, Kinney C, Lin C, Jan D, Moore G, Betenbaugh MJ. 2009. Expression of anti-apoptosis genes alters lactate metabolism of Chinese Hamster Ovary cells in culture.Biotechnology and Bioengineering $103: 592-608$.

8. Droste P, Miebach S, Niedenführ S, Wiechert W, Nöh K. 2011. Visualizing multi-omics data in metabolic networks with the software Omix - A case study. Biosystems 105 :154-161.

9. Estes S, Melville M. 2013. Mammalian Cell Line Developments in Speed and Efficiency. In: . Springer, Berlin, Heidelberg, pp. 11-33. http://link.springer.com/10.1007/10_2013_260.

10. Fontova A, Lecina M, López-Repullo J, Martínez-Monge I, Comas P, Bragós R, Cairó JJ. 2018. A simplified implementation of the stationary liquid mass balance method for on-line OUR monitoring in animal cell cultures. Journal of Chemical Technology and Biotechnology 93 .

11. Fussenegger M, Fassnacht D, Schwartz R, Zanghi JA, Graf M, Bailey JE, Pörtner R. 2000. Regulated overexpression of the survival factor bcl-2 in CHO cells increases viable cell density in batch culture and decreases DNA release in extended fixed-bed cultivation. Cytotechnology 32 :45-61.

12. Grilo AL, Mantalaris A. 2019. The Increasingly Human and Profitable Monoclonal Antibody Market. Trends in Biotechnology $37: 9-16$.

13. Hartley F, Walker T, Chung V, Morten K. 2018. Mechanisms driving the lactate switch in Chinese hamster ovary cells. Biotechnology and Bioengineering $115: 1890-1903$.

14. Henry MN, MacDonald MA, Orellana CA, Gray PP, Gillard M, Baker K, Nielsen LK, Marcellin E, Mahler S, Martínez VS. 2020. Attenuating apoptosis in Chinese hamster ovary cells for improved 
biopharmaceutical production. Biotechnology and Bioengineering117:1187-1203.

15. Henry O, Durocher Y. 2011. Enhanced glycoprotein production in HEK-293 cells expressing pyruvate carboxylase. Metabolic Engineering $13: 499-507$.

16. Hnasko RM, Stanker LH. 2015. Hybridoma Technology. In: Hnasko, R, editor. ELISA . New York, NY: Springer New York, Vol. 1318, pp. 15-28. http://link.springer.com/10.1007/978-1-4939-2742-5_2.

17. Juanola S, Vives J, Milián E, Prats E, Cairó JJ, Gòdia F. 2009. Expression of BHRF1 improves survival of murine hybridoma cultures in batch and continuous modes. Applied Microbiology and Biotechnology $83: 43-57$.

18. Lecina M, Soley A, Gràcia J, Espunya E, Lázaro B, Cairó JJJ, Gòdia F. 2006. Application of on-line OUR measurements to detect actions points to improve baculovirus-insect cell cultures in bioreactors. Journal of Biotechnology $125: 385-394$.

19. Lewis NE, Hixson KK, Conrad TM, Lerman JA, Charusanti P, Polpitiya AD, Adkins JN, Schramm G, Purvine SO, Lopez-Ferrer D, Weitz KK, Eils R, König R, Smith RD, Palsson BØ. 2010. Omic data from evolved E. coli are consistent with computed optimal growth from genome-scale models. Molecular Systems Biology 6 :390.

20. Majors BS, Betenbaugh MJ, Chiang GG. 2007. Links between metabolism and apoptosis in mammalian cells: applications for anti-apoptosis engineering. Metab. Eng. $9: 317-326$.

21. Majors BS, Betenbaugh MJ, Pederson NE, Chiang GG. 2009. Mcl-1 overexpression leads to higher viabilities and increased production of humanized monoclonal antibody in Chinese hamster ovary cells.Biotechnol. Prog. $25: 1161-1168$.

22. Martínez VS, Dietmair S, Quek L-E, Hodson MP, Gray P, Nielsen LK. 2013. Flux balance analysis of $\mathrm{CHO}$ cells before and after a metabolic switch from lactate production to consumption. Biotechnology and Bioengineering $110: 660-666$.

23. Martínez-Monge I, Albiol J, Lecina M, Liste-Calleja L, Miret J, Solà C, Cairó JJ. 2018a. Metabolic Flux Balance Analysis during lactate and glucose concomitant consumption in HEK293 cell cultures.Biotechnology and Bioengineering . http://doi.wiley.com/10.1002/bit.26858.

24. Martínez-Monge I, Comas P, Triquell J, Casablancas A, Lecina M, Paredes C, Cairó JJ. 2019a. Concomitant consumption of glucose and lactate: A novel batch production process for $\mathrm{CHO}$ cells.Biochemical Engineering Journal :107358.

25. Martínez-Monge I, Comas P, Triquell J, Lecina M, Casablancas A, Cairó JJJ. 2018b. A new strategy for fed-batch process control of HEK293 cell cultures based on alkali buffer addition monitoring: comparison with O.U.R. dynamic method. Applied Microbiology and Biotechnology . http://www.ncbi.nlm.nih.gov/pubmed/302 http://link.springer.com/10.1007/s00253-018-9388-4.

26. Martínez-Monge I, Roman R, Comas P, Fontova A, Lecina M, Casablancas A, Cairó JJ. 2019b. New developments in online OUR monitoring and its application to animal cell cultures. Appl Microbiol Biotechnol103:6903-6917.

27. Mastrangelo AJ, Hardwick JM, Zou S, Betenbaugh MJ. 2000. Part II. Overexpression of bcl-2 family members enhances survival of mammalian cells in response to various culture insults. Biotechnol. Bioeng. $67: 555-564$.

28. McKeehan WL. 1986. Glutaminolysis in Animal Cells. In: Morgan, MJ, editor. Carbohydrate Metabolism in Cultured Cells . Boston, MA: Springer US, pp. 111-150. https://doi.org/10.1007/978-1-46847679-8_4.

29. Mulukutla BC, Gramer M, Hu W-S. 2012. On metabolic shift to lactate consumption in fed-batch culture of mammalian cells. Metabolic Engineering $14: 138-149$.

30. Quek L-E, Dietmair S, Krömer JO, Nielsen LK. 2010. Metabolic flux analysis in mammalian cell culture. Metabolic Engineering $12: 161-171$.

31. Quek L-E, Nielsen LK. 2008. On the reconstruction of the Mus musculus genome-scale metabolic network model. In: . Genome Informatics 2008: Genome Informatics Series Vol. 21 . World Scientific, pp. 89-100.

32. Robergs RA, Ghiasvand F, Parker D. 2004. Biochemistry of exercise-induced metabolic acidosis. Am. J. Physiol. Regul. Integr. Comp. Physiol. 287 :R502-516. 
33. Rocha I, Maia P, Evangelista P, Vilaça P, Soares S, Pinto JP, Nielsen J, Patil KR, Ferreira EC, Rocha M. 2010. OptFlux: an open-source software platform for in silico metabolic engineering. BMC Systems Biology $4: 45$.

34. Sauerwald TM, Oyler GA, Betenbaugh MJ. 2003. Study of caspase inhibitors for limiting death in mammalian cell culture.Biotechnol. Bioeng. 81 :329-340.

35. Schantz PG, Sjöberg B, Svedenhag J. 1986. Malate-aspartate and alpha-glycerophosphate shuttle enzyme levels in human skeletal muscle: methodological considerations and effect of endurance training. Acta Physiol. Scand. $128: 397-407$.

36. Sheikh K, Förster J, Nielsen LK. 2005. Modeling Hybridoma Cell Metabolism Using a Generic GenomeScale Metabolic Model of Mus musculus. Biotechnology Progress 21 :112-121.

37. Simpson NH, Milner AE, Al-Rubeai M. 1997. Prevention of hybridoma cell death by bcl-2 during suboptimal culture conditions. Biotechnol. Bioeng. 54 :1-16.

38. Smolke CD. 2010. The metabolic pathway engineering handbook : fundamentals. CRC Press/Taylor \& Francis.

39. Templeton N, Lewis A, Dorai H, Qian EA, Campbell MP, Smith KD, Lang SE, Betenbaugh MJ, Young JD. 2014. The impact of anti-apoptotic gene Bcl-2 $\Delta$ expression on $\mathrm{CHO}$ central metabolism. Metabolic Engineering 25 :92-102.

40. Tey BT, Singh RP, Piredda L, Piacentini M, Al-Rubeai M. 2000. Influence of Bcl-2 on cell death during the cultivation of a Chinese hamster ovary cell line expressing a chimeric antibody.Biotechnology and Bioengineering $68: 31-43$.

41. Valgepea K, Loi KQ, Behrendorff JB, Lemgruber R de SP, Plan M, Hodson MP, Köpke M, Nielsen LK, Marcellin E. 2017. Arginine deiminase pathway provides ATP and boosts growth of the gas-fermenting acetogen Clostridium autoethanogenum. Metabolic Engineering 41 :202-211.

42. Vives J, Juanola S, Cairó JJ, Prats E, Cornudella L, Gòdia F. 2003a. Protective Effect of Viral Homologues of bcl-2 on Hybridoma Cells under Apoptosis-Inducing Conditions. Biotechnology Progress 19 :84-89.

43. Vives J, Juanola S, Cairó JJ, Gòdia F. 2003b. Metabolic engineering of apoptosis in cultured animal cells: implications for the biotechnology industry. Metabolic Engineering 5 :124-132.

44. Walsh G. 2018. Biopharmaceutical benchmarks 2018. Nat Biotechnol 36 :1136-1145.

45. Wurm FM. 2004. Production of recombinant protein therapeutics in cultivated mammalian cells. Nat Biotechnol22:1393-1398.

46. Xiong K, Marquart KF, Karottki KJ la C, Li S, Shamie I, Lee JS, Gerling S, Yeo NC, Chavez A, Lee GM, Lewis NE, Kildegaard HF. 2019. Reduced apoptosis in Chinese hamster ovary cells via optimized CRISPR interference. Biotechnology and Bioengineering 116 :1813-1819.

47. Yang M, Butler M. 2000. Effects of ammonia on CHO cell growth, erythropoietin production, and glycosylation. Biotechnology and bioengineering $68: 370-380$.

48. Yoon S, Konstantinov KB. 1994. Continuous, real-time monitoring of the oxygen uptake rate (OUR) in animal cell bioreactors.Biotechnology and Bioengineering 44 :983-990.

49. Zagari F, Jordan M, Stettler M, Broly H, Wurm FM. 2013. Lactate metabolism shift in CHO cell culture: the role of mitochondrial oxidative activity. New Biotechnology 30 :238-245.

50. Zhang L, Shen H, Zhang Y. 2004. Fed-batch culture of hybridoma cells in serum-free medium using an optimized feeding strategy. Journal of Chemical Technology \& Biotechnology79:171-181.

51. Zhang X, Han L, Zong H, Ding K, Yuan Y, Bai J, Zhou Y, Zhang B, Zhu J. 2018. Enhanced production of anti-PD1 antibody in CHO cells through transient co-transfection with anti-apoptotic genes BclxLand Mcl-1.Bioprocess Biosyst Eng 41 :633-640.

52. Zheng J. 2012. Energy metabolism of cancer: Glycolysis versus oxidative phosphorylation (Review). Oncology Letters 4 :1151-1157.

53. Zhou W, Hu W-S. 1994. On-line characterization of a hybridoma cell culture process. Biotechnology and Bioengineering $44: 170-177$.

\section{TABLES}




\section{Nomenclature}

\begin{tabular}{lll}
\hline $\boldsymbol{\mu}$ & Specific growth rate & {$\left[\mathrm{h}^{-1}\right]$} \\
\hline $\mathbf{O U R}$ & Oxygen uptake rate & {$[\mathrm{mmol} /(\mathrm{L} \cdot \mathrm{h})]$} \\
$\mathbf{q}_{\mathbf{x}}$ & Specific consumption or production of metabolite $\mathbf{x}$ & {$[\mathrm{nmol} /(\mathrm{mg} \cdot \mathrm{h})]$} \\
$\mathbf{T C A}$ & Cycle of tricarboxylic acids & - \\
$\mathbf{t}_{\mathbf{d}}$ & Duplication time & {$[\mathrm{h}]$} \\
$\mathbf{V C D}$ or $\mathbf{X}_{\mathbf{V}}$ & Viable cell concentration & {$\left[10^{6} \mathrm{cell} / \mathrm{mL}\right]$} \\
$\mathbf{Y}_{\mathbf{G l u} / \mathbf{X}}$ & Yield of glucose with respect to biomass & {$\left[(\mathrm{mmol} / \mathrm{L}) /\left(10^{6} \mathrm{cell} / \mathrm{mL}\right)\right]$} \\
$\mathbf{Y}_{\mathbf{L a c} / \mathbf{X}}$ & Yield of lactic with respect to biomass & {$\left[(\mathrm{mmol} / \mathrm{L}) /\left(10^{6} \mathrm{cell} / \mathrm{mL}\right]\right.$} \\
$\mathbf{q}_{\mathbf{P}}$ & Specific productivity & {$\left[\mu \mathrm{g} /\left(10^{6} \mathrm{cell} \cdot \mathrm{h}\right)\right]$} \\
$\mathbf{V}_{\mathbf{P}}$ & Volumetric productivity & {$[(\mu \mathrm{g} / \mathrm{L}) / \mathrm{h}]$} \\
$\left.[\mathbf{P r o d u c t}]_{\text {final }}\right]$ & Final product concentration & {$[\mu \mathrm{g} / \mathrm{L}]$} \\
$\mathbf{I C V}$ & Integral of viable cell concentration & {$\left[10^{6} \mathrm{cell} / \mathrm{mL} \cdot \mathrm{h}\right]$} \\
$\mathbf{t}_{\text {total }}$ & Total process time before the viability starts to decay & {$[\mathrm{h}]$} \\
$\mathbf{m A b}$ & Monoclonal antibody & \\
\hline
\end{tabular}

Table 1. Main physiological parameters determined for KB26.5 and KB26.5-BHRF1 in shake flasks cultures. Values have been calculated as the mean of the data obtained from duplicates cultures.

\begin{tabular}{lll}
\hline Parameter & KB26.5 & KB26.5-BHRF1 \\
\hline$\mu\left(\mathrm{h}^{-1}\right)$ & $0.027 \pm 0.001$ & $0.048 \pm 0.002$ \\
$\mathrm{t}_{\mathrm{d}}(\mathrm{h})$ & $25.500 \pm 0.928$ & $14.560 \pm 0.627$ \\
$\mathrm{q}_{\mathrm{Glu}}(\mathrm{nmol} /(\mathrm{mg} \cdot \mathrm{h}))$ & $-802.325 \pm 71.750$ & $-682.100 \pm 55.536$ \\
$\mathrm{q}_{\mathrm{Lac}}(\mathrm{nmol} /(\mathrm{mg} \cdot \mathrm{h}))$ & $1337.750 \pm 175.857$ & $663.780 \pm 13.831$ \\
$\mathrm{Y}_{\mathrm{Glu} / \mathrm{X}}(\mathrm{mmol} / \mathrm{L}) /\left(10^{6} \mathrm{cell} / \mathrm{mL}\right)$ & $14.515 \pm 0.120$ & $4.645 \pm 0.007$ \\
$\mathrm{Y}_{\mathrm{Lac} / \mathrm{X}}(\mathrm{mmol} / \mathrm{L}) /\left(10^{6} \mathrm{cell} / \mathrm{mL}\right)$ & $24.265 \pm 1.039$ & $5.035 \pm 1.704$ \\
$\mathrm{VCD}_{\max }\left(10^{6} \mathrm{cell} / \mathrm{mL}\right)$ & $1.79 \pm 0.30$ & $3.92 \pm 0.03$ \\
\hline
\end{tabular}

Table 2 . Main physiological parameters determined for KB26.5 and KB26.5-BHRF1 cultures run in 2-Litres bioreactor. Values have been calculated as the mean of the data obtained from duplicates cultures.

\begin{tabular}{|c|c|c|}
\hline Parameter & KB26.5 & KB26.5-BHRF1 \\
\hline$\overline{\mu\left(h^{-1}\right)}$ & $0.036 \pm 0.006$ & $0.048 \pm 0.007$ \\
\hline $\mathrm{t}_{\mathrm{d}}(\mathrm{h})$ & $19.823 \pm 3.588$ & $14.593 \pm 2.001$ \\
\hline $\mathrm{q}_{\mathrm{Glu}}(\mathrm{nmol} /(\mathrm{mg} \cdot \mathrm{h}))$ & $-933.447 \pm 134.567$ & $-542.206 \pm 177.457$ \\
\hline $\mathrm{q}_{\mathrm{Lac}}(\mathrm{nmol} /(\mathrm{mg} \cdot \mathrm{h}))$ & $1435.500 \pm 348.745$ & $654.556 \pm 34.663$ \\
\hline $\mathrm{Y}_{\mathrm{Glu} / \mathrm{X}}(\mathrm{mmol} / \mathrm{L}) /\left(10^{6} \mathrm{cell} / \mathrm{mL}\right)$ & $8.805 \pm 0.361$ & $3.735 \pm 0.346$ \\
\hline $\mathrm{Y}_{\mathrm{Lac} / \mathrm{X}}(\mathrm{mmol} / \mathrm{L}) /\left(10^{6} \mathrm{cell} / \mathrm{mL}\right)$ & $14.010 \pm 0.665$ & $4.730 \pm 1.160$ \\
\hline $\mathrm{VCD}_{\max }\left(10^{6} \mathrm{cell} / \mathrm{mL}\right)$ & $2.34 \pm 0.39$ & $3.93 \pm 0.01$ \\
\hline $\operatorname{IgG}_{3, \max }(\mathrm{mg} / \mathrm{L})$ & $12.90 \pm 3.82$ & $47.70 \pm 2.69$ \\
\hline $\mathrm{q}_{\mathrm{P}}\left(\mu \mathrm{g} /\left(10^{6} \mathrm{cell} \cdot \mathrm{h}\right)\right)$ & $0.24 \pm 0.08$ & $0.48 \pm 0.02$ \\
\hline $\mathrm{V}_{\mathrm{P}}((\mu \mathrm{g} / \mathrm{L}) / \mathrm{h})$ & $252.12 \pm 74.63$ & $750.22 \pm 0.85$ \\
\hline
\end{tabular}

Table 3 . Specific consumption rates of glucose, amino acids and oxygen, and production rates of lactate and ammonia for KB26.5 and KB26.5-BHRF1 cell lines. Cultures were run in 2-Litres bioreactors. Specific rates are part of the 38 external measured fluxes used as a modeling constrains and are express in $(\mathrm{nmol} /(\mathrm{mg} \cdot \mathrm{h}))$ 
units.

\begin{tabular}{lll}
\hline Parameter $\left(\mathbf{n m o l} / \mathbf{m g} \cdot \mathbf{h}^{-\mathbf{1}}\right)$ & KB26.5 & KB26.5-BHRF1 \\
\hline Alanine & $132.493 \pm 12.954$ & $48.305 \pm 4.158$ \\
Arginine & $-14.726 \pm 2.812$ & $-15.517 \pm 8.825$ \\
Asparagine & $5.420 \pm 1.302$ & $3.058 \pm 0.681$ \\
Aspartic acid & $4.304 \pm 6.017$ & $0.487 \pm 0.801$ \\
Biomass & $0.036 \pm 0.006$ & $0.048 \pm 0.007$ \\
Cysteine & $-5.024 \pm 2.111$ & $-4.825 \pm 4.079$ \\
Glucose & $-933.447 \pm 134.567$ & $-542.206 \pm 177.457$ \\
Glutamic acid & $7.858 \pm 0.265$ & $0.841 \pm 0.956$ \\
Glutamine & $-258.541 \pm 76.627$ & $-179.660 \pm 122.421$ \\
Glycine & $-11.597 \pm 6.102$ & $-12.094 \pm 6.784$ \\
Histidine & $-6.744 \pm 3.693$ & $-5.368 \pm 3.796$ \\
Isoleucine & $-24.989 \pm 5.918$ & $-18.098 \pm 16.359$ \\
Lactate & $1435.500 \pm 348.745$ & $654.556 \pm 34.663$ \\
Leucine & $-28.854 \pm 6.149$ & $-24.593 \pm 17.043$ \\
Lysine & $-22.759 \pm 9.314$ & $-21.579 \pm 17.480$ \\
Methionine & $-7.315 \pm 0.948$ & $-6.511 \pm 4.841$ \\
NH ${ }_{4}^{+}$ & $156.137 \pm 11.064$ & $85.090 \pm 15.910$ \\
Phenylalanine & $-9.685 \pm 2.050$ & $-10.009 \pm 8.848$ \\
Proline & $13.080 \pm 4.084$ & $1.993 \pm 8.586$ \\
Oxygen & $-701.500 \pm 160.513$ & $-674.500 \pm 12.021$ \\
Serine & $-15.150 \pm 6.181$ & $-13.913 \pm 11.826$ \\
Threonine & $-16.883 \pm 3.216$ & $-18.522 \pm 18.500$ \\
Tryptophan & $-2.585 \pm 3.297$ & $-1.078 \pm 0.722$ \\
Tyrosine & $-8.340 \pm 1.832$ & $-8.392 \pm 7.974$ \\
Valine & $-24.539 \pm 4.938$ & $-20.236 \pm 18.013$ \\
\hline
\end{tabular}

\section{FIGURES}

Figure 1 . Time profile of viable cell density $\left({ }^{(}\right.$, cellviability $(*)$, glucose $(*)$ andlactate () concentrationofbatchculturesruninsh parentalKB26.5andB1 : engineeredKB26.5-BHRF1). Napierianlogarithmofviablecelldensityversustime $(*)(A 2$ : parentalK B26.5andB2 : engineeredK B26.5-BHRF1).Dataobtainedfromduplicatesculturesinshakeflasksandtheerrorba

Figure 2 . 2-Litres bioreactor batch cultures of the Parental KB26.5 cell line (A.1 and A.2) and the engineered KB26.5-BHRF1 (B1 and B2). Time profile of cell density $\left({ }^{(}\right.$, cellviability $(*) ;$ glucose $(*)$, lactate ()andproduct(bars) conc

Figure 3 . 2-Litres bioreactor batch cultures of the Parental KB26.5 cell line (A.1 and A.2) and the engineered KB26.5-BHRF1 (B1 and B2). Napierian logarithm of viable cell density versus time $(*)$. Duplicate cultures are presented for each cell line.

Figure 4 . Metabolic fluxes distribution of parental KB26.5 hybridoma cell line. The arrows indicate the direction of the reaction and the arrow's thickness is proportional to the flux rate. Different coloring has been used for each pathway and model was structured in cytoplasmic and mitochondrial reactions (mitochondrion is limited by a discontinued line). The value therein the boxes corresponds to the flux rate in (nmol metabolite $/(\mathrm{mg} \mathrm{DCW} * \mathrm{~h}))$.

Figure 5 . Metabolic fluxes distribution of the engineered hybridoma cell line KB26.5-BHRF1. The arrows indicate the direction of the reaction and the arrow's thickness is proportional to the flux rate. Different coloring has been used for each pathway and model was structured in cytoplasmic and mitochondrial reactions (mitochondrion is limited by a discontinued line). The value therein the boxes corresponds to the flux rate in (nmol metabolite/ mg DCW*h). 
Figure 6. Distribution of ATP generation (concerning the reaction in which ATP is consumed and generated in both cell lines KB26.5 and KB26.5-BHRF1. ATP consumption (A) and production (B) (nmol ATP/ (mg $\left.\mathrm{DCW}^{*} \mathrm{~h}\right)$ ).
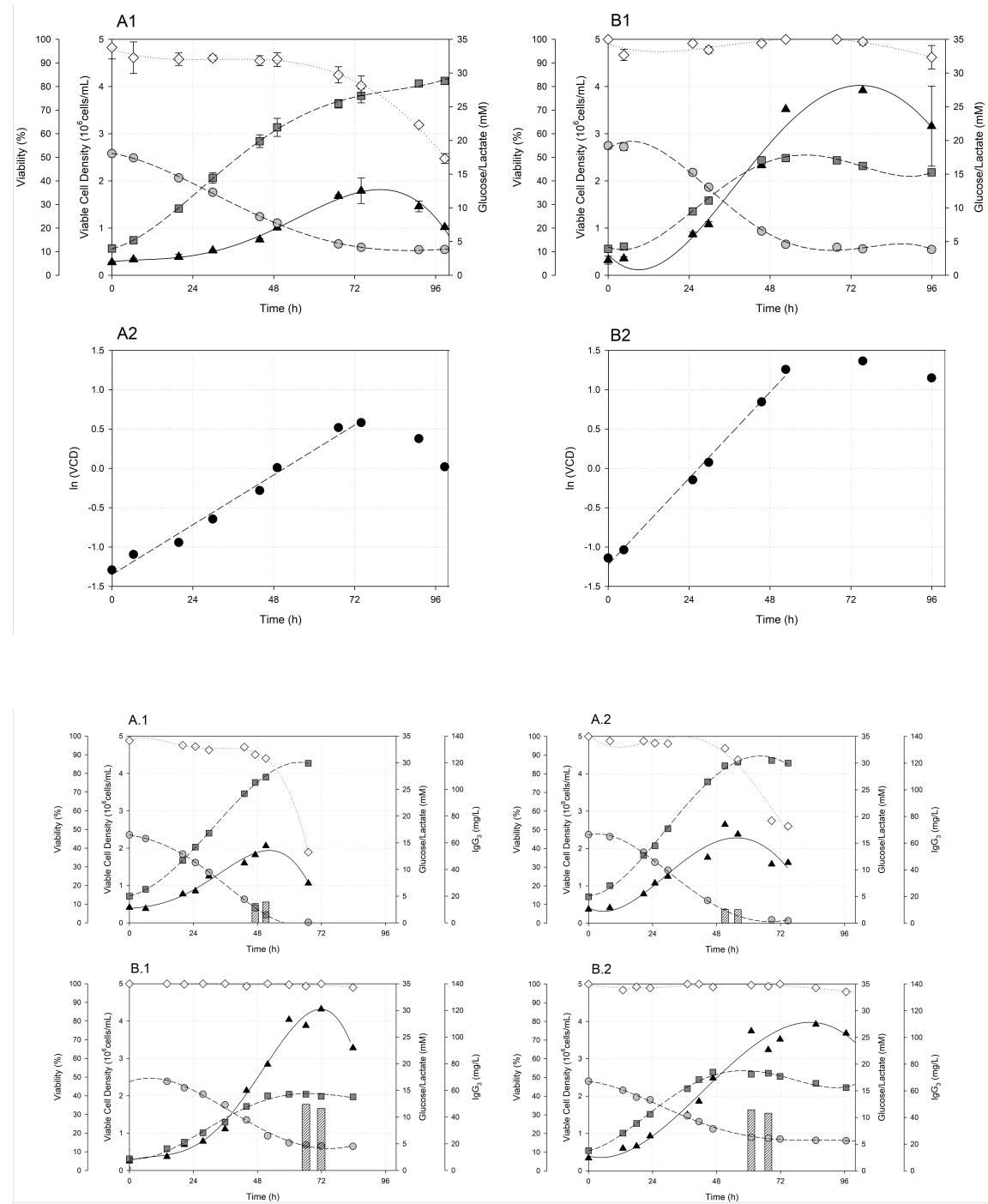

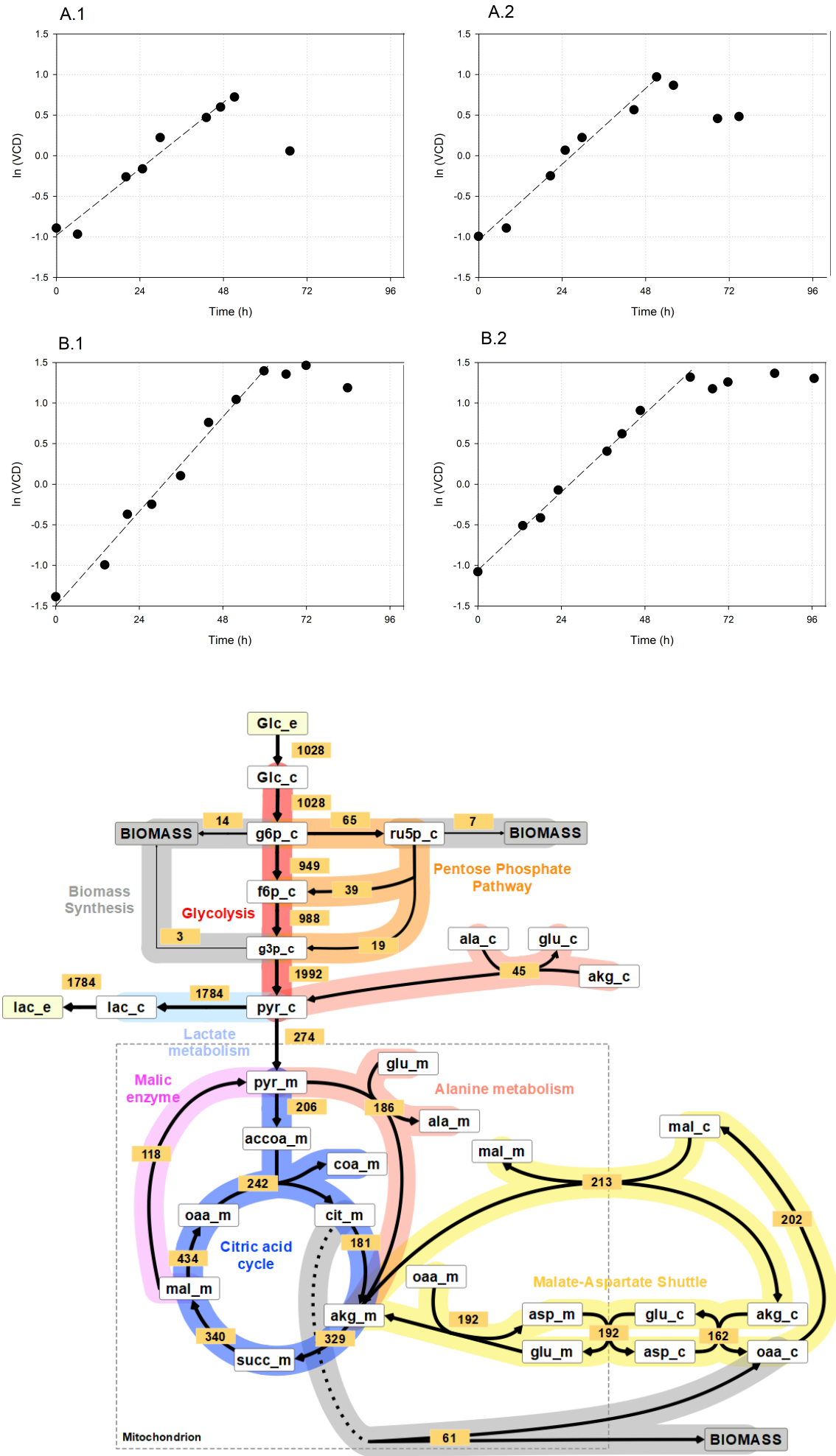


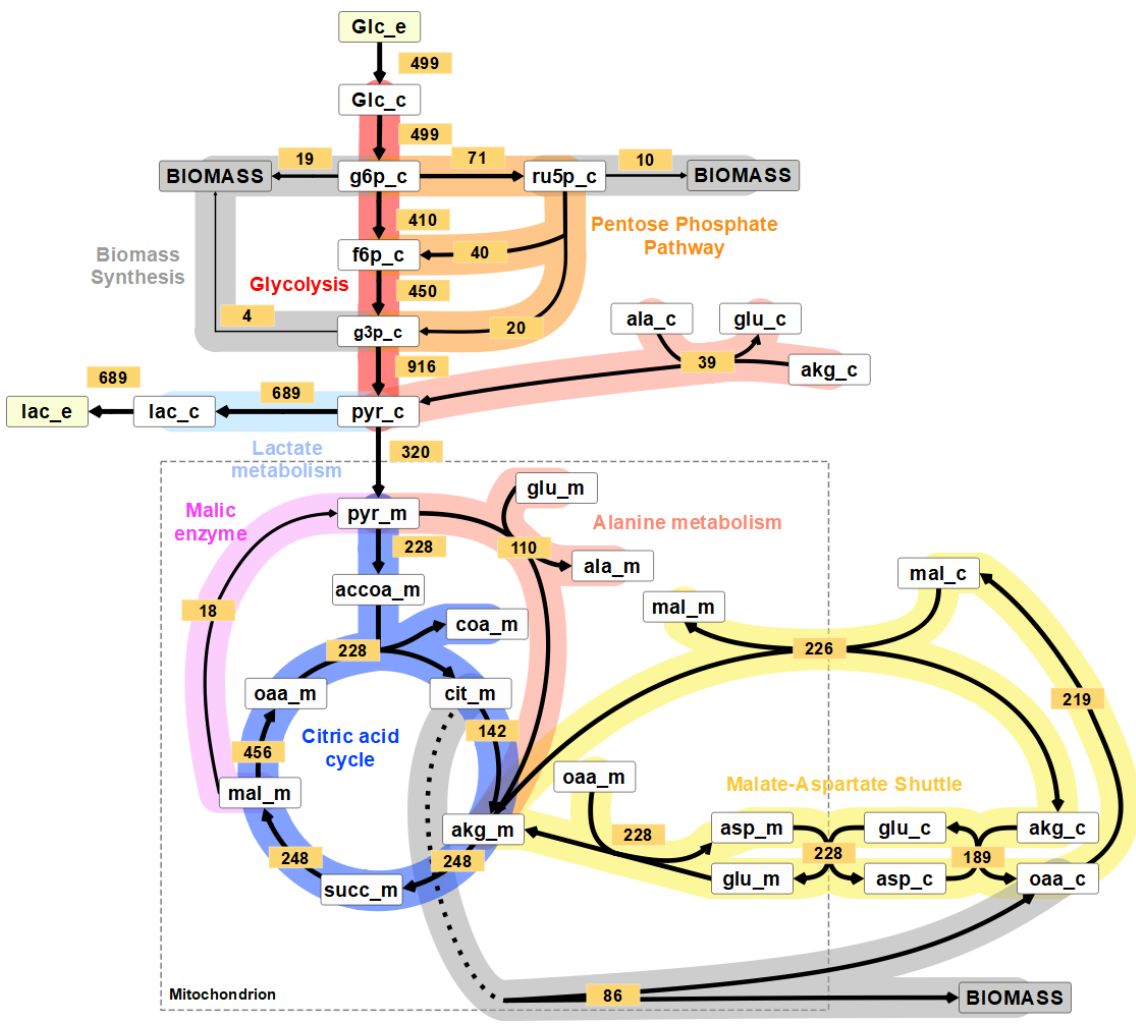



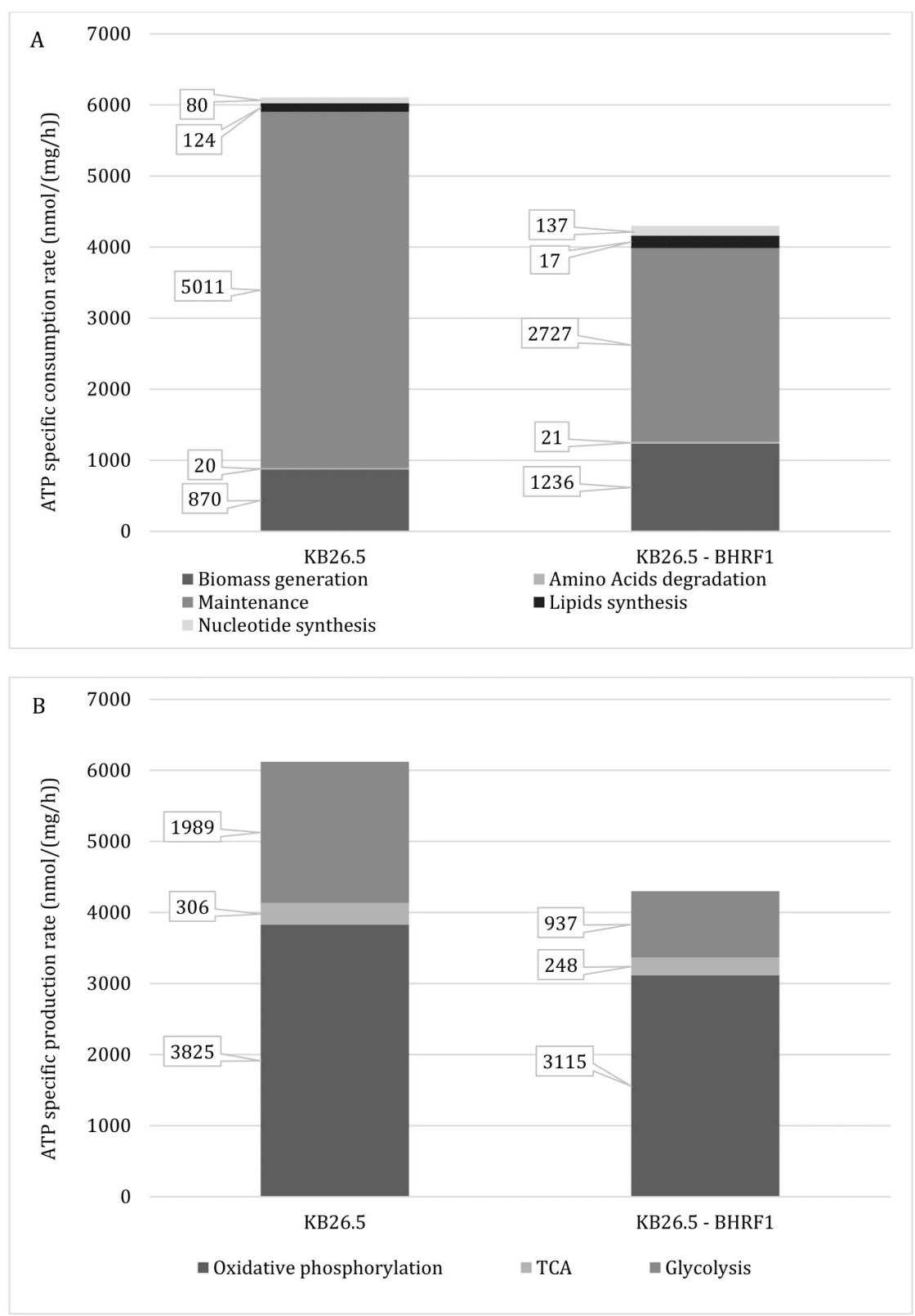